Acta Crystallographica Section A

Foundations of Crystallography

ISSN 0108-7673

Received 14 May 2012

Accepted 23 October 2012

C 2013 International Union of Crystallography Printed in Singapore - all rights reserved

\section{Propagation of neutron spherical waves through a thick, vibrating Ge single crystal}

\author{
E. Raitman, ${ }^{a *}$ V. Gavrilov, ${ }^{\text {a }}$ D. Mjasishchev, ${ }^{a}$ A. Hoser, ${ }^{b}$ O. Seidel ${ }^{b}$ and J. Stanh ${ }^{c}$ \\ anstitute of Physical Energetics, Riga, Latvia, ${ }^{\mathbf{b}}$ Helmholtz-Zentrum, BENSC, Berlin, Germany, and \\ 'Paul Scherrer Institute, Villigen, Switzerland. Correspondence e-mail: mdmitry@delfi.lv
}

\begin{abstract}
The results of experimental and theoretical studies into the influence of ultrasound on the propagation of neutron waves in a thick Ge crystal are presented. The neutron intensity profiles were measured for the case of Laue diffraction inside the Borrmann fan. At low amplitudes of ultrasonic waves interference effects (diffraction intensity beatings) were observed. The observations were possible because of the uniform acoustic-field distribution through the whole bulk of the crystal. As distinct from the classical Shull experiments, wide analysing slits or position-sensitive detectors were used. To explain the results obtained, a modified theory for the spatial distribution of neutron diffraction intensities in the presence of acoustic excitation of the crystal is proposed. A good agreement between experiment and theory is obtained. At high amplitudes of ultrasonic waves the transition to kinematic scattering was not observed, despite the large strains in the crystalline lattice created by ultrasound. This could be connected with the formation of a superlattice having a standing wave period. A strong rise in the diffraction intensity and a sharp constriction of the neutron beam at the centre of the Borrmann fan were observed. This new effect could be used for the creation of ultrasound-controlled monochromators.
\end{abstract}

\section{Introduction}

Experiments confirming the correctness of theoretical considerations based on the dynamical theory of wave radiation fields in the interior of a perfect crystal were performed by Kato (Kato \& Lang, 1959; Kato \& Ando, 1966; Kato, 1969) for X-ray and later by Shull (Shull, 1968, 1973; Zeilinger et al., 1983) for neutron diffraction in silicon and germanium single crystals. The theoretical description of dynamical diffraction considers the neutron wave pattern propagating in the periodic potential of an ideal lattice. This theory explains a variety of diffraction features such as, for example, intensity Pendellösung oscillations, intensity variations within a Borrmann fan, anomalous transmission of X-rays and neutrons etc. One of the most important effects of dynamical diffraction is the strongly limited intensity diffracted by an ideal crystal in the Bragg position. In the case of Laue diffraction, the distribution of the diffracted intensities has an oscillating form within a Borrmann fan (Rauch \& Petrashek, 1979; Abov et al., 2002). However, the interference effects disappear very quickly if the translation symmetry in a perfect crystal is violated due to any disturbance, such as static deformation strain or lowfrequency sound excitations (Entin, 1979). In this case, the Bragg-reflected intensity increases, and neutron diffraction is described as a rule by the kinematical theory of radiation scattering.
The purpose of this paper is to present experimental and theoretical results of the study of acoustic wave (AW) distortions or dynamic strain effects in the propagation of neutron waves. The neutron intensity profiles for the case of Laue diffraction in the interior of the Borrmann fan, i.e. the pattern of neutron distribution at the exit of the crystal for a thick Ge crystal, were measured. Our experiments presented here, on the neutron Bragg scattering in a Ge single crystal excited by a high-frequency transverse standing $\mathrm{AW}$, are a version of Shull's experiments. However, our experiments were carried out with monochromatic neutrons and a perfect crystal disturbed by ultrasound, instead of Shull's 'imperfect' neutrons (a white beam with high collimation) and perfect crystals. Another distinction from Shull's classical experiments is the use of position-sensitive detectors (PSDs) instead of very narrow analysing slits $[0.13 \mathrm{~mm}$ (Shull, 1973) or $0.1 \mathrm{~mm}$ (Abov et al., 2002)].

Ultrasonic waves are a simple and well defined disturbance of the crystal lattice, and allow one to study the transition in diffraction from a perfect crystal reflecting a weak intensity towards an imperfect crystal diffracting an enlarged neutron wavelength band. The degree of crystal imperfection is easily controlled by the sound-wave amplitude (Kulda et al., 1988; Michalec et al., 1988; Hock et al., 1998).

In the case of neutron wave propagation in a temporally and spatially modulated crystal the interference of Bloch 
waves depends on the ultrasound wave amplitude, while neutron diffraction is well described by the dynamical theory of scattering, even at very strong and periodical distortion of the crystalline lattice.

Recently, the intensities of neutron Bragg diffraction from the front and back faces of a thick, perfect Ge single crystal undergoing ultrasound excitation have been measured and calculated theoretically (Raitman et al., 2010). It was shown that, simultaneously with the AW amplitude increasing, the main Bragg peaks grow and the back-face peaks become asymmetric and tend to disappear. Such back-face scattering was observed for the first time. This effect is successfully described within the frameworks of the dynamical theory of neutron scattering modified in the presence of ultrasound excitation and Kato's quasi-classical approximation taking into account the new ultrasonic extinction length.

The main aim of our work was the search for spatial oscillations of the diffracted neutron intensity for the case of weak ultrasound excitations and their analytical treatment. This effect was predicted theoretically (Iolin \& Entin, 1983). Also, it has been shown that high-frequency ultrasound mixes the states corresponding to the various sheets of the dispersion surface (Iolin et al., 1986). This mixing leads to a number of new effects, e.g. the resonant suppression of the Borrmann effect (Entin, 1979) and radiation intensity Pendellösung dependent on the acoustic wave amplitude (Entin \& Puchkova, 1984; Iolin et al., 1986).

Below, a spatial Pendellösung of the neutron intensities observed for the first time near the centre of the Borrmann fan on a thick Ge single crystal is described and analysed. For a description of the experimental data obtained, the modified theory of the spatial distribution of diffracted neutrons in the presence of a crystal ultrasonic excitation is offered. A good agreement between experiment and theory is obtained.

In addition, it is shown that strong ultrasound leads to the appearance of an intense, narrow neutron beam at the centre of the Borrmann fan. This effect could be used for the creation of ultrasound-controlled monochromators.

\section{Experimental}

\subsection{Experimental layout}

The experiments were carried out using an HZB BENSC E6 diffractometer $\left(\lambda_{\mathrm{n}}=0.243 \mathrm{~nm}\right)$, a V12a double-crystal diffractometer $\left(\lambda_{\mathrm{n}}=0.471 \mathrm{~nm}\right)$ and a PSI MORPHEUS diffractometer $\left(\lambda_{\mathrm{n}}=0.410 \mathrm{~nm}\right)$. The experimental layout is shown in Fig. 1. The sample was a Ge single crystal [(111) reflection] with dimensions $V=52 \times 22 \times 20 \mathrm{~mm}=Y \times L \times$ $T \mathrm{~mm}$, where $Y$ is the sample length, $T$ is the sample thickness and $L$ is the distance on which the standing AW propagation was taken. A monochromatic and well collimated neutron beam with a cross section of $1 \times 10 \mathrm{~mm}$ was directed to the sample. The sample quality was checked preliminarily and the FWHM of the rocking curve was 3.1", close to the theoretical expectation. For the observation of the diffracted neutron distribution two techniques were used. One of them used an analysing slit $B$ (Fig. 1), similar to the classic experiments but with a width of $0.5 \mathrm{~mm}$ - different to the $0.1 \mathrm{~mm}$ slits used by Shull (1973) and Abov et al. (2002) for intensity oscillation observations - to avoid 'non-sound' intensity oscillations (Shull fringes). In the other method an analysing slit was not used. Instead, the spatial distribution of the reflected neutron beam was measured with a PSD. After finding the optimal relation $\theta-2 \theta$ (adjustment), the sample and PSD were kept motionless and the reflected intensity distribution was directly measured in $2 \theta$; the coordinates were then recalculated for the current $x$ coordinates (E6 and V12a diffractometers). This procedure in general is similar to the scanning with slit method; however, it allows much faster data acquisition.

\subsection{Acoustic field in the sample}

The transverse AW excitation was realized using a quartz piezo transducer with main frequency $v_{s}=41.3 \mathrm{MHz}$. The polarized AW propagated perpendicular to the scattering vector $H\left(k_{s} \perp H, u \| H\right)$, where $u$ is the displacement of a nucleus for standing transverse waves excited between the two parallel surfaces of the sample with waves amplitude $w$. The piezo transducer was glued to the sample using salol. As shown below, the diffraction intensity is proportional to $|H w|$; therefore its values could be used for estimation of the acoustic field inside the crystal (Fig. 2). Owing to the diffractive divergence of acoustic waves (Truell et al., 1972), this field is concentrated not only in the region around the piezo transducer but is distributed uniformly enough through the whole sample, especially at the levels of weak and moderate excitation. It was more important to check the uniformity of a sound field in the Borrmann fan. In these experiments the slit

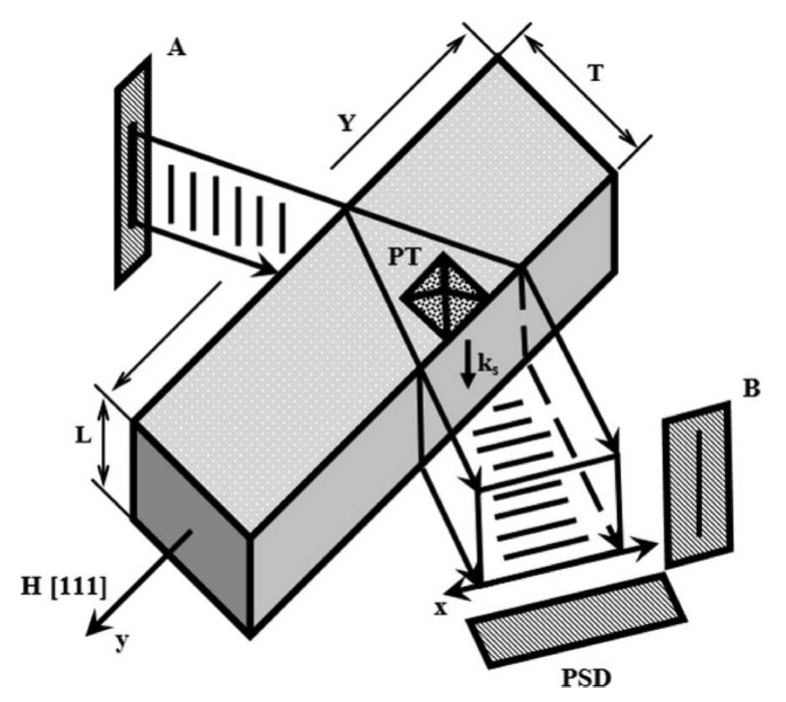

Figure 1

The layout of the experiment: $A$ and $B$ are the forming and analysing slits, respectively; PSD is the position-sensitive detector; PT is the piezo transducer; $H$ is the vector of the reciprocal lattice; $k_{s}$ is the AW wavenumber; $y$ is the direction of the sample movement (slit $B$ is motionless); $x$ is the current coordinate in the base of the Borrmann fan and the direction of movement of slit $B$. In this case, the sample is motionless. 


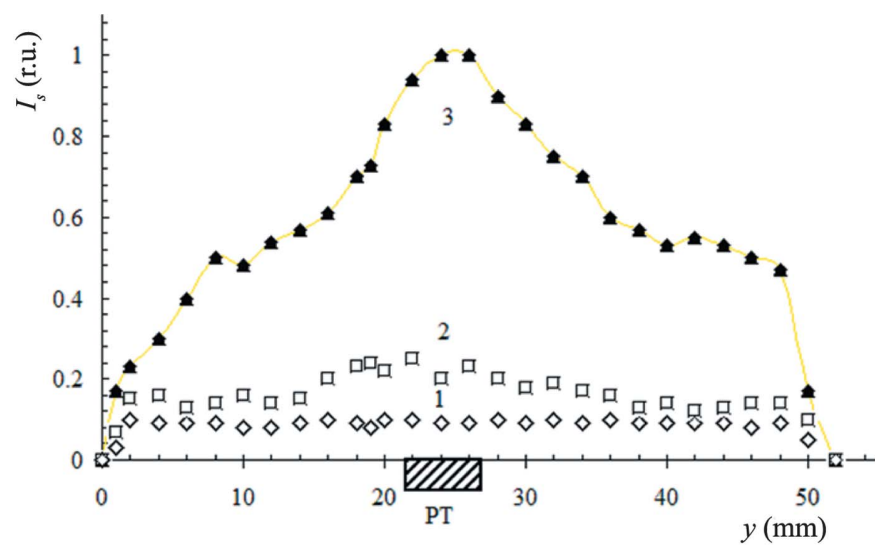

Figure 2

Diffraction intensity distribution in relative units at different sample displacements along the $y$ axis: (1) in the absence of ultrasonic excitation; (2) $V_{\mathrm{G}}=0.3 \mathrm{~V}$; (3) $V_{\mathrm{G}}=5 \mathrm{~V}$. PT is the piezo transducer. The $I_{s}$ value is proportional to the AW amplitude.

$B$ remained motionless and the sample was moved in the direction $y$ with a step of $2 \mathrm{~mm}$.

The uniformity of the acoustic-field distribution within a Borrmann fan is an important prerequisite to ensure observation of the spatial oscillations of diffraction intensity. Assume that in the diffraction process a finite volume of the sample participates, in which the amplitude $w$ of AW oscillations is distributed in the interval $w \pm \Delta w$. From the theoretical expression $\Delta k_{s}=\Delta K_{0}|H w|$ (Iolin \& Entin, 1983; Iolin et al., 1986), it follows that a spread in wave amplitude $w$ is connected to a spread in wavevector $k_{s}$. This leads to a smearing of the pulse gap $\left(k_{s} \pm \Delta k_{s}\right)$; therefore the phase difference of the corresponding Bloch waves on the crystal surface will be $\Delta \varphi=\Delta k_{s} T=\Delta K_{0} H T(w \pm \Delta w)$. Since the intensity oscillations are approximately described by the function $I \simeq \cos ^{2}\left(\Delta k_{s} T\right)$, the $n$th intensity maximum corresponds to the condition $\Delta \varphi=\Delta k_{0} H w T=n \pi$ where $n$ is an oscillation number. At a phase shift of $\Delta \varphi_{1}=\pi / 2$ the maximum of diffracted intensity $I_{s}$ becomes its minimum. Therefore, to observe oscillations it is necessary that the phase shift $\Delta \varphi_{1}$ is less than $\pi / 2$. From this follows the estimation $\Delta w / w<1 /(2 n)$. At a reasonable distribution of $w$ values $(\Delta w / w \simeq 10 \%)$ this leads to the situation where in the experiment only the first few oscillations can be observed. From the data shown in Fig. 2 (curve 2) it is seen that the conditions $\Delta w / w \leq 10 \%$ are fulfilled well at low amplitudes of the AW.

\subsection{Velocities of the acoustic standing waves}

As a rule, theoretical estimations of the AW effect on the diffraction intensity were made for the case of coherent sound. As follows from Fig. 3(a), in our experiment there is a wide enough frequency spectrum of excitations described by a Gaussian and the AW cannot be considered as a single mode. However, as was previously shown (Iolin et al., 1996), the assumption of sound coherence is not always necessary for the correct interpretation of the experimental results. When

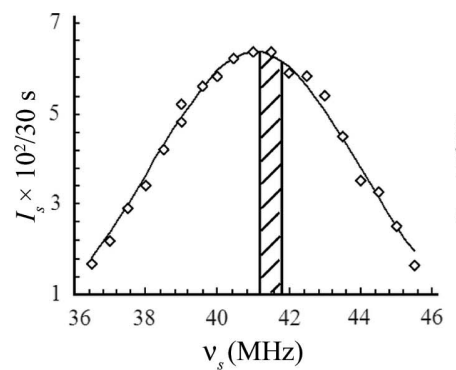

(a)

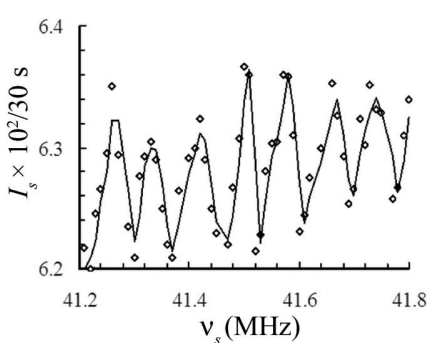

(b)
Figure 3

Dependence of the Laue diffraction intensity $I_{s}$ at the Borrmann fan centre on the ultrasound frequency $\left(\lambda_{\mathrm{n}}=0.410 \mathrm{~nm}, S=1 \mathrm{~mm}\right)$ : $(a)$ the frequency scanning step is $0.5 \mathrm{MHz}$; the solid curve is a result of data fitting by a Gaussian with an FWHM of $6.34 \mathrm{MHz}$; the region in which the detailed frequency scanning was carried out is shaded $(41.2-41.8 \mathrm{MHz})$; (b) the same as in $(a)$ only for the shaded frequency range, shown in $(a)$. The frequency scanning step is $\sim 0.01 \mathrm{MHz}$; the solid curve was obtained by averaging over three data points (spline approximation).

the diffraction intensity depends on the frequency under scanning with a smaller step (Fig. 3b), a fine structure appears which is evidence of the presence of an acoustic standing wave in the crystal. For acoustic standing waves the relation $L=i \lambda_{s} / 2$ should be fulfilled, where $i$ is an integer of halfwaves, $\lambda_{s}$ is the AW wavelength and $L=22 \mathrm{~mm}$ is the distance of AW propagation. If we know $L$ and the distance between the maxima (minima) of the fine structure of the frequency dependence $\Delta v_{s}=\left[v_{s i}-v_{s(i \pm 1)}\right]$ (Fig. $\left.3 b\right)$, it is possible to determine the velocity of sound propagation. Determining $\Delta v_{s}=(0.08 \pm 0.01) \mathrm{MHz}$ from the data in Fig. 3(b), we obtain $i=v_{s i} / \Delta v=517-524$ (with an accuracy of $\sim 10 \%$ ) and $v_{s}=$ $(3.52 \pm 0.20) \times 10^{5} \mathrm{~cm} \mathrm{~s}^{-1}$. This value is very close to the reference value of $3.55 \times 10^{5} \mathrm{~cm} \mathrm{~s}^{-1}$ (McSkimin, 1950) for the velocity of a shear AW for a Ge single crystal in the [100] direction.

\section{Main results and discussion}

\subsection{Theoretical background}

The effect of an AW on neutron diffraction in a perfect crystal is shown schematically in Fig. 4 . The new energy gaps $k_{s}$

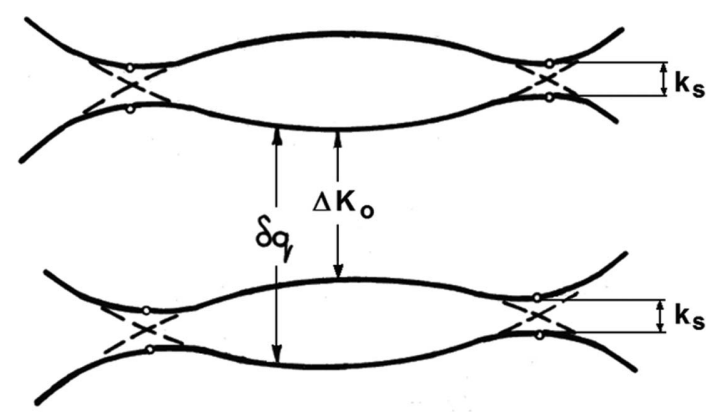

Figure 4

Schematic image of the dispersion surface modified by ultrasound (Laue geometry) in a perfect crystal. $\Delta K_{0}$ is the gap between the sheets of the dispersion surface determining the extinction length $\left(\tau=2 \pi / \Delta K_{0}\right)$; and $k_{s}$ are the new AW energy gaps determining the additional regions of Bragg reflection (Iolin \& Entin, 1983; Iolin et al., 1986). 
appear on dispersion surfaces which correspond to the additional regions of total reflection for the case of Bragg reflection. The AW phonon's absorption (emission) by neutrons mixes Bloch states and displaces the dispersion surface of the neutron by the value of the quasi-momentum $\delta q$ :

$$
\delta q=v_{s} /\left(v_{\mathrm{n}} \cos \theta_{\mathrm{B}}\right) \pm k_{s} .
$$

The neutron-acoustic resonance frequency $v_{\text {res }}$ is determined by equation (2) (Iolin \& Entin, 1983; Raitman et al., 2009):

$$
v_{\text {res }}=\left(v_{\mathrm{n}} \cos \theta_{\mathrm{B}}\right) / \tau,
$$

where $\theta_{\mathrm{B}}$ and $v_{\mathrm{n}}$ are the Bragg angle and the neutron velocity, respectively, and $\tau$ is the extinction length. Equation (1) is valid if $H \perp k_{s}$ and this corresponds to our experimental conditions (Fig. 1). The resonance frequency $v_{\text {res }}$ is equal to $19.6 \mathrm{MHz}$ for $\mathrm{Ge}(111)$ and, taking into account the DebyeWaller factor, its value does not depend on the observed reflection (Iolin et al., 1996; Raitman et al., 2009). For the case $v_{s}>v_{\text {res }}$ a linear increase in the diffraction intensity should be observed depending on the AW amplitude $w$ (Iolin et al., 1986).

The change in the diffracted neutron intensity distribution for the crystal by the AW can be interpreted qualitatively and quantitatively by the dynamical diffraction theory. Calculations taking into consideration the strains created by the AW which affect the neutron wavefield inside the crystal can be made using modified Takagi-Taupin equations (Takagi, 1962; Taupin, 1961; Iolin et al., 1988):

$$
\begin{array}{r}
i \alpha \frac{\partial \Psi_{0}}{\partial t}=-i \frac{\partial \Psi_{0}}{\partial z}-i \tan \theta_{\mathrm{B}} \frac{\partial \Psi_{0}}{\partial x}+\frac{\Delta k_{0}}{2} \exp (i H u) \Psi_{\mathrm{h}} \\
i \alpha \frac{\partial \Psi_{\mathrm{h}}}{\partial t}=-i \frac{\partial \Psi_{\mathrm{h}}}{\partial z}+i \tan \theta_{\mathrm{B}} \frac{\partial \Psi_{\mathrm{h}}}{\partial x}+\frac{\Delta k_{0}}{2} \exp (-i H u) \Psi_{0},
\end{array}
$$

where $\alpha=1 / v_{\mathrm{n}} \cos \theta_{\mathrm{B}}$ and $u=w \sin (\omega t) \sin \left(k_{s} z\right)$ is the displacement of a nucleus for standing transverse waves excited between the two parallel surfaces of the sample with wave amplitude $w$, wavelength $\lambda_{s}$ and wavenumber $k_{s}=$ $2 \pi / \lambda_{s} . \Psi_{0}, \Psi_{\mathrm{h}}$ are the amplitudes of the incident and diffracted beams, respectively, $v_{\mathrm{n}}$ is the velocity of the neutron wave and $\Delta k_{0}=2 \pi / \tau$ with $\tau$ being the extinction length. Solving the set of equations (3) and (4) we can determine the diffraction intensity at the centre of the Borrmann fan for the plane monochromatic neutron wave approximation depending on $w$, $k_{s}, \lambda_{\mathrm{n}}$ and $\tau$. However, such a determination is not precise enough for calculation of the spatial distribution of the diffraction intensity - even near the centre of the Borrmann fan, when the whole dispersive surface is excited.

\subsection{Spatial intensity distribution of the diffracted neutron beam}

Owing to the interference of Bloch waves, the distribution of diffracted neutron beam intensity in a crystal arising on its outside surface has characteristic beats at the centre and the intensity increases towards the edges of the Borrmann fan. These effects are described by equation (5) (Kato, 1969; Abov et al., 2002):

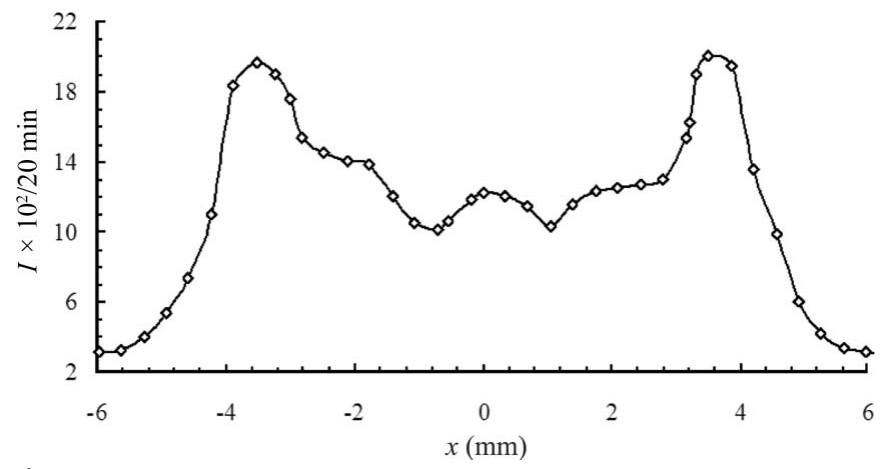

Figure 5

Normalized intensity distribution for an Si single crystal [reflection (111)], $B$-slit width $S \leq 0.2 \mathrm{~mm}, T=11 \mathrm{~mm}$. Neutron wavelength $\lambda_{\mathrm{n}}=0.1 \mathrm{~nm}$. The so-called Shull's fringe is clearly seen at the centre of the profile (Kuvaldin, 1991).

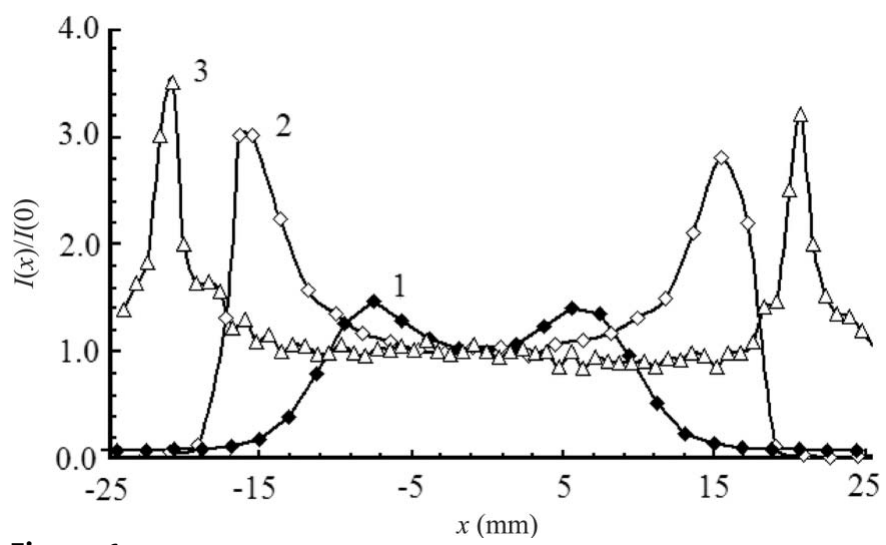

Figure 6

Normalized intensity distribution for reflection (111) of Ge $(T=20 \mathrm{~mm})$ (Borrmann fan) for different neutron wavelengths: (1) $\lambda_{\mathrm{n}}=0.471 \mathrm{~nm}$; (2) $\lambda_{\mathrm{n}}=0.410 \mathrm{~nm}$; (3) $\lambda_{\mathrm{n}}=0.243 \mathrm{~nm}$. Curve 2 was obtained using a slit with $S$ $=1 \mathrm{~mm}$. Curves 1 and 3 were obtained without an analysing slit (PSD only).

$$
I_{0}(\Gamma)=c\left(1-\Gamma^{2}\right)^{-1 / 2} \cos ^{2}\left[A\left(1-\Gamma^{2}\right)^{-1 / 2}\right],
$$

where $\Gamma=x /\left(2 T \tan \theta_{\mathrm{B}}\right)$ is the deviation of a neutron wave from the atomic plane trace, $A=\pi T / \tau, c$ is a normalization constant and $x$ is the current coordinate in the base of the Borrmann fan. Equation (5) is valid for the diffraction of a plane monochromatic wave in the symmetrical Laue case. A more rigorous expression for the shape of the reflected beam distribution can be obtained with the help of a spherical neutron wave approximation (Kato, 1969):

$$
I_{0}(\Gamma)=(A / 2) J_{0}^{2}\left[A\left(1-\Gamma^{2}\right)^{1 / 2}\right]
$$

where $J_{0}$ is the Bessel function of zero order. According to equations (5) and (6), the intensity of a diffracted beam oscillates on the outside surface of a crystal, with the oscillation period depending on the parameter $\pi T / \tau$, and decreases quickly outward from the centre of profile $(\Gamma=0)$ (Figs. 5 and 6).

The diffraction intensity distribution shown in Fig. 6 (curve 2) was obtained using an analysing slit $B$ with width $S=1 \mathrm{~mm}$. Curves 1 and 3 were observed with no slit at all, employing instead a high-resolution PSD. In both cases the oscillations are averaged, and the dependence of the full size of the 
Table 1

Comparison of calculated and experimental data for the spatial distribution of intensities for different $\lambda_{\mathrm{n}}$.

$S$ is the slit width. $T$ is the sample thickness. Experimental results for $\mathrm{Si}(111)$ are taken from Kuvaldin (1991; Fig. 5). The $X_{\text {theor }}$ values calculated by equation (7) coincide well with the experimental ones.

\begin{tabular}{lllccll}
\hline$T(\mathrm{~mm})$ & $\begin{array}{l}\lambda_{\mathrm{n}} \\
(\mathrm{nm})\end{array}$ & $\begin{array}{l}S \\
(\mathrm{~mm})\end{array}$ & $\begin{array}{l}X_{\text {theor }} \\
(\mathrm{mm})\end{array}$ & $\begin{array}{l}X_{\text {exp }} \\
(\mathrm{mm})\end{array}$ & $I(\Gamma=1) / I(\Gamma=0)$ & $I(\Gamma=-1) / I(\Gamma=0)$ \\
\hline $\mathrm{Si}(111), 11 \mathrm{~mm}$ & 0.101 & 0.2 & 7.04 & 7.2 & 1.64 & 1.61 \\
& & & & & & 1.40 \\
$\mathrm{Ge}(111), 20 \mathrm{~mm}$ & 0.243 & PSD & 15.22 & 15.0 & 1.46 & 2.80 \\
& 0.410 & 1.0 & 30.61 & 31.5 & 3.00 & 3.22 \\
& 0.471 & PSD & 39.34 & 40.2 & 3.50 & \\
\hline
\end{tabular}

$$
\begin{aligned}
I_{\text {in }}= & (H w)^{2}\left[1+\frac{\delta q^{2}}{\left(\delta q^{2}-\Delta K_{0}^{2}\right)}\right] J_{0}^{2} \\
& \times\left[A H w(1-\Omega)^{1 / 2}\right],
\end{aligned}
$$

where

$$
\Omega=\frac{\Gamma^{2} \delta q^{2}}{\left(\delta q^{2}-\Delta K_{0}^{2}\right)}, \quad A=\pi T / \tau
$$

Equations (9) and (10) describe the central part of a Borrmann fan:

Borrmann fan base $X$ on the neutron wavelength follows from the averaged equation (5) (Table 1):

$$
X=\frac{2 T \lambda_{\mathrm{n}}}{\left(4 d^{2}-\lambda_{\mathrm{n}}^{2}\right)^{1 / 2}} \text {. }
$$

\subsection{High-frequency sound effect on the spatial distribution of the diffracted neutron intensity}

The presence of new energy gaps $k_{s}$ (Fig. 4) on the dispersion surface under ultrasound should lead to the appearance of an additional structure in the intensity distribution of diffracted neutrons at the exit of a crystal. The new distribution, as shown below, depends on the AW amplitude, $w$, while 'sound' oscillations are superimposed on the initial ones. The size of the first soundless oscillation for the Ge (111) reflection in the vicinity of $\Gamma=0$ according to equation (5) is $\Delta x_{1}=$ $(T \tau)^{1 / 2} \tan \theta_{\mathrm{B}} \simeq 1.5 \mathrm{~mm}$. The numerical analysis of experimental data based on the known theoretical expressions involves difficulties, since they have been obtained in the plane neutron wave approximation. Such an approximation cannot be applied to the description of the intensity distribution of diffracted neutrons on the Borrmann fan base when the whole dispersion surface is excited.

We have derived expressions for the diffraction intensity of a spherical neutron wave in an ideal crystal under ultrasound excitation. In this case the total diffraction intensity $I_{\mathrm{t}}$ is composed of the elastic and inelastic components:

$$
\begin{gathered}
I_{\mathrm{t}}=I_{\mathrm{el}}+I_{\mathrm{in}} \\
I_{\mathrm{el}}=I_{0}+\Delta I_{\mathrm{el}},
\end{gathered}
$$

where $I_{\text {in }}$ and $\Delta I_{\mathrm{el}}$ are the elastic and inelastic additions, respectively; $I_{0}$ is determined from equation (6), while the elastic addition to the diffraction intensity is given by equation (9):

$$
\Delta I_{\mathrm{el}}=\frac{2 \Delta K_{0}^{2}(H w)^{2} \sin ^{2}(\delta q T / 2) J_{1}^{2}\left[A H w(1-\Omega)^{1 / 2}\right]}{\left(\delta q^{2}-\Delta K_{0}^{2}\right)(1-\Omega)}
$$

where $J_{1}$ is the Bessel function of the first order and $\delta q$ is the displacement of the dispersive surface at the absorption or emission of an ultrasound phonon. The main contribution to the intensity of inelastic (one-phonon) scattering is made by the term

$$
-\left(1-\Delta K_{0}^{2} / \delta q^{2}\right)^{1 / 2} \leq \Gamma \leq\left(1-\Delta K_{0}^{2} / \delta q^{2}\right)^{1 / 2} ;
$$

these are valid at $\Delta K_{0} H w=\left(\delta q-\Delta K_{0}\right)$, which corresponds to the weak interaction of satellites with the main Bragg maximum. At $v_{s} \gg v_{\text {res }},\left(\delta q \gg \Delta K_{0}\right)$, i.e. under the conditions of our experiment, $I_{\mathrm{in}}$ far exceeds $\Delta I_{\mathrm{el}}$. This was previously confirmed by our experiments with measuring the spin-echo in a silicon single crystal under ultrasound pumping (Iolin et al., 1998).

The spatial distribution of diffraction intensity is described by the expression

$$
I_{s}(\Gamma, H w)=I_{0}+I_{\mathrm{in}},
$$

where $I_{\text {in }}$ is taken in the form of equation (10) and $I_{0}$ of equation (6). The character of the spatial distribution of the diffraction intensity is dictated by three parameters: $\mathrm{A}, \mathrm{Hw}$ and the $\delta q / \Delta K_{0}$ ratio. The choice of a thick $(T \simeq 70 \tau) \mathrm{Ge}$ crystal is not quite optimal, since the first AW oscillations will appear when the 'acoustic' extinction length is $\tau_{s}=\tau|H w|^{-1}$, i.e. the degree of crystal perfection should be very high. On the other hand, a thick crystal provides a long enough Borrmann fan, which allows for easy and detailed measurements of the spatial distribution of the diffracted beam intensity (Fig. 7).

Already at a small amplitude of the acoustic wave $H w=0.26$ $(w=0.013 \mathrm{~nm})$, the diffraction intensity in the centre starts rising noticeably. The width of the first oscillation (Fig. 7) on the $x$ axis (close to $\Gamma=0$ ) at $H w=0$ could be estimated from equation (5): $\Delta x_{1}=(2 T \tau)^{1 / 2} \tan \theta_{\mathrm{B}}$ and $\Delta x_{1} \simeq 6 \mathrm{~mm}$ for the Ge (111) reflection. Since at the appearance in the vicinity of $\Gamma=0$ of a new 'sound' extinction length $\tau_{s}=\tau|H w|^{-1}$, at $H w \ll 1$ the linear size of the first 'sound' fringe will increase as $\Delta x_{s}=\Delta x_{1}|H w|^{-1 / 2}$. As follows from Fig. 7, at $H w=0.26$ the size of the first acoustic fringes is $11.4 \mathrm{~mm}$, which coincides well with the calculated value of $12 \mathrm{~mm}$. Besides, at least the next two oscillations could be clearly discerned. As $H w$ further increases, at the profile centre a linear intensity rise is observed. To compare the theoretical and experimental data a high-precision determination of the $A$ and $H w$ parameters is needed, since the Bessel function oscillates quickly at large values of the argument. The calculated $A$ value is 2071 , i.e. close to $A=2060$, at which 'soundless' distribution of the diffraction intensity $(H w=0)$ is described most satisfactorily by equation (6), taking into account integration over the width of the analysing slits and correct data averaging. The AW 

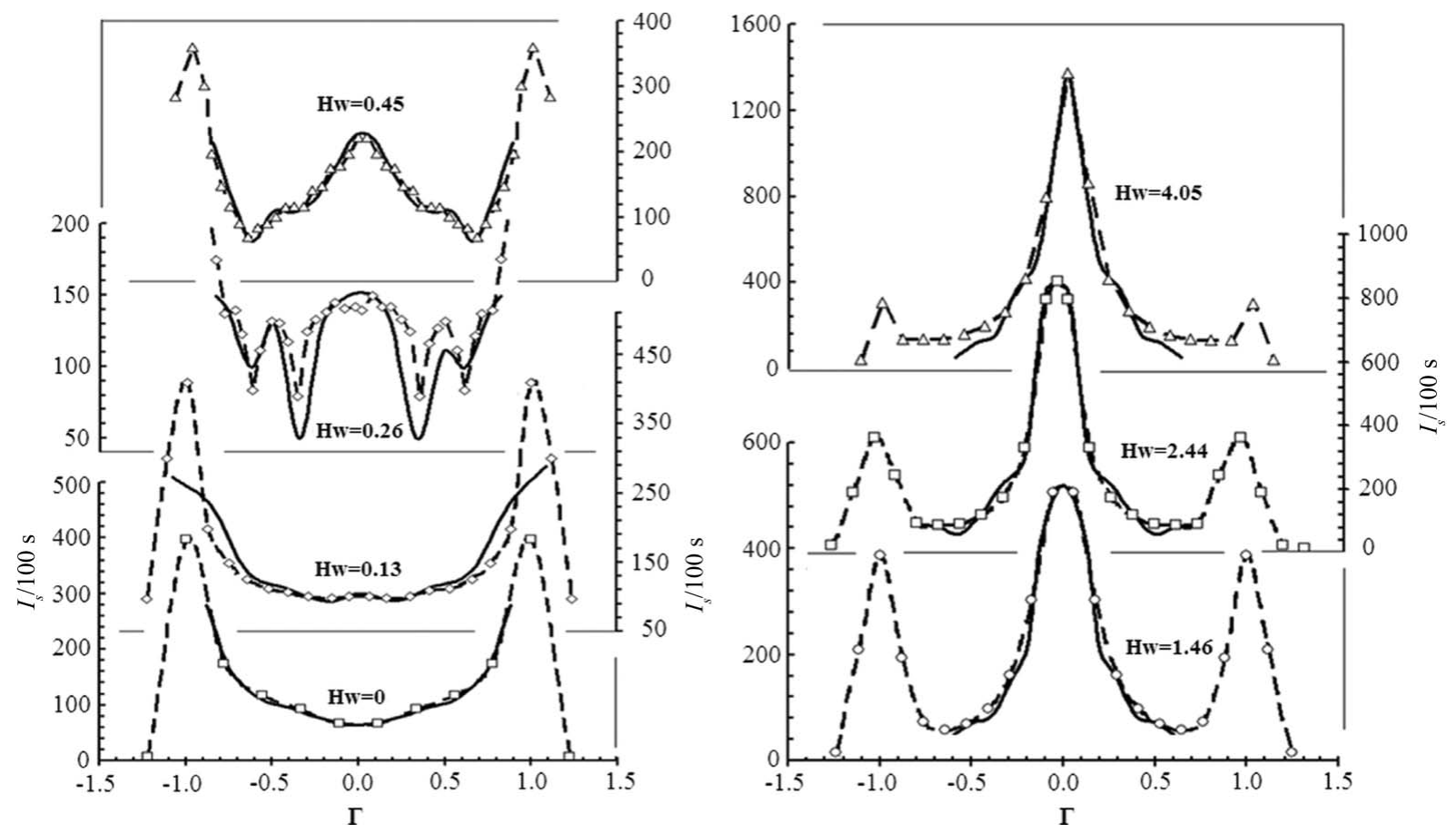

Figure 7

Spatial distribution of a diffracted beam intensity versus the AW amplitude $w$ and $\Gamma$ value. Dashed curves represent averaged experimental data, solid curves are a result of data fitting by equations (10)-(12).

amplitude can be determined by changes in relative intensity variations at the centre of the spatial profile $(\Gamma=0)$ :

$$
\eta=\frac{I_{s}-I_{0}}{I_{0}}=\frac{I_{\text {in }}}{I_{0}}=\frac{(H w)^{2}\left[1+\delta q^{2} /\left(\delta q^{2}-\Delta K_{0}^{2}\right)\right] J_{0}^{2}(H w A)}{J_{0}^{2}(A)} .
$$

At large values of the argument $J_{0}(z) \rightarrow$ $(2 / \pi z)^{1 / 2} \cos (z-\pi / 4)$, the relative contribution of the neutron inelastic scattering by AW oscillations of the lattice with a frequency above resonant is

$$
\eta(H w)=4 H w \frac{\cos ^{2}(H w A)}{\cos ^{2}(A)} \simeq 4 H w .
$$

The experimental data shown in Fig. 8 were obtained for the same sample but on different neutron diffractometers using different RF generators and wide-band amplifiers. Besides, every time the piezo transducer was reglued anew. However, in all cases a linear dependence of $\eta$ on the generator voltage $V_{\mathrm{G}}$ was observed, which makes it possible to find a calibration constant $C$ and to determine the AW amplitude from the relationship

$$
\eta=C V_{\mathrm{G}}=|H w| .
$$

The effect of saturation was observed in the $\eta$ dependence versus $V_{\mathrm{G}}$ for large $H w$. The heating of the organic glue leads to a decrease in its viscosity and brings about violation of the proportionality between $V_{\mathrm{G}}$ and $w$. This fact was taken into account and the measurements were considered reliable up to $H w \leq 3.5$.
Fig. 9 shows the spatial distribution of diffraction intensity $I_{s}$ depending on the acoustic wave amplitude for neutrons of different wavelength. Independently of the neutron wavelength, $I_{s}$ at the Borrmann fan centre increases with increasing $H w$.

Fig. 10 demonstrates a sharp decrease in the spatial halfwidth of the central peaks which is dependent on the AW amplitude. This result is unexpected, since in some earlier works where the possibility of creating ultrasound-driven/ controlled monochromators was discussed (Guigay et al., 1990; Mikula et al., 1992; Hock et al., 1993) the gain in intensity with rising AW amplitude is always compensated by a loss in resolution (the FWHM rises). In contrast, in our experiments

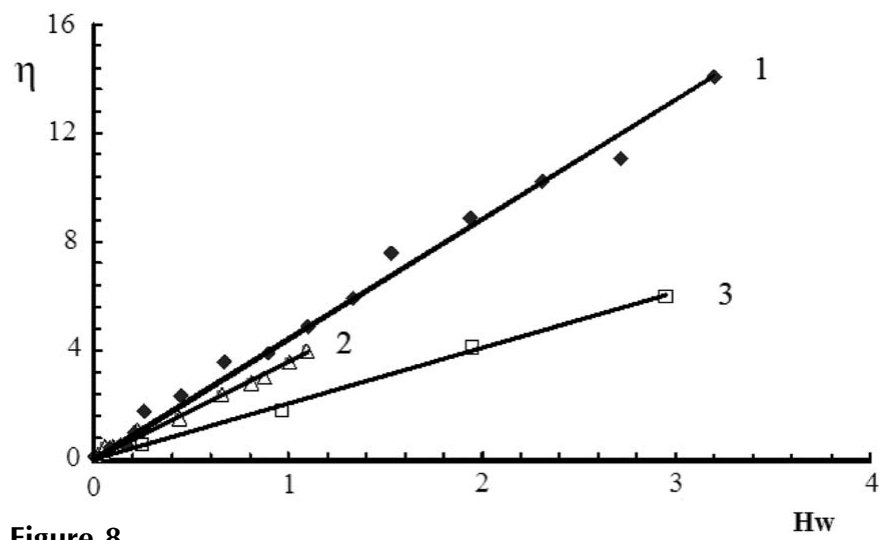

Figure 8 Hw

Diffraction intensity at the centre of a Borrmann fan $(\Gamma=0)$ versus AW amplitude for neutrons of different wavelength: (1) $\lambda_{\mathrm{n}}=0.41 \mathrm{~nm}$; (2) $\lambda_{\mathrm{n}}=$ $0.471 \mathrm{~nm}$; (3) $\lambda_{\mathrm{n}}=0.243 \mathrm{~nm}$. Solid curves: fitting taking into account equations (13)-(15). 


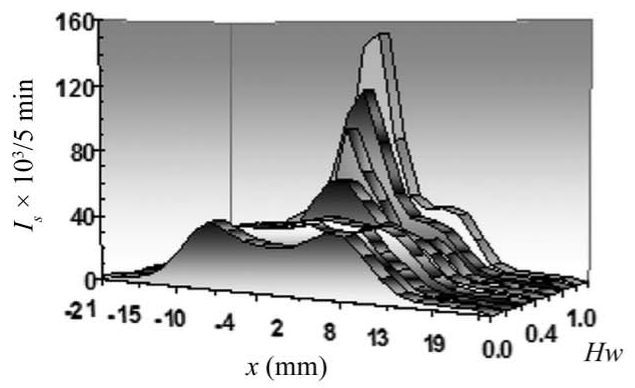

(a)

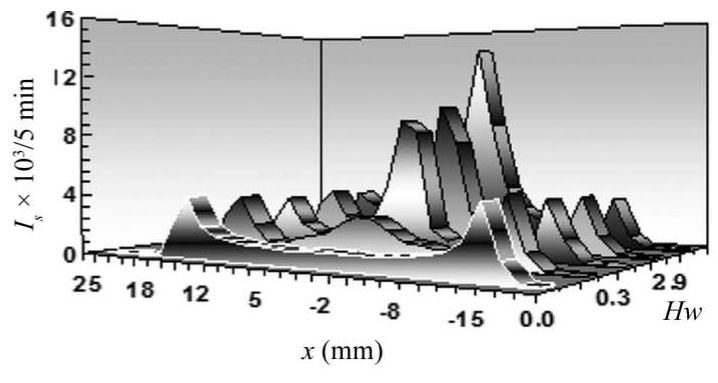

(b)

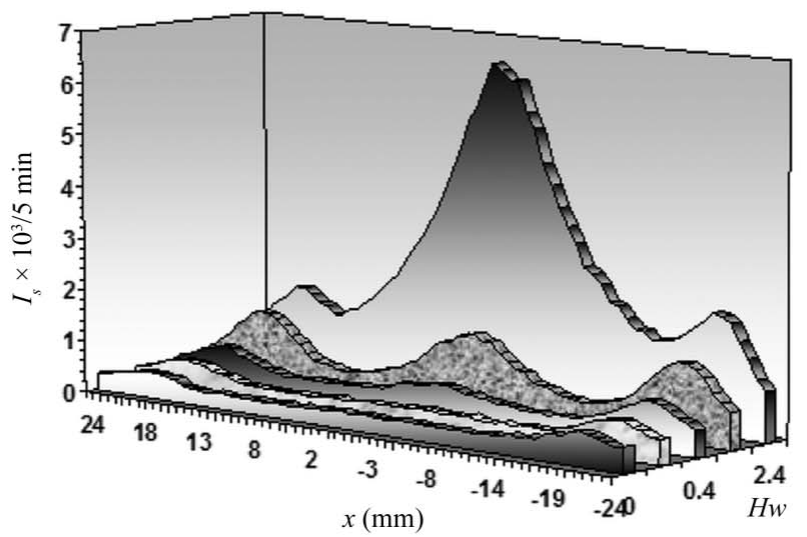

(c)

Figure 9

The neutron diffraction intensity as a function of acoustic wave amplitudes $H w$ and running coordinate $x$ in the base of the Borrmann fan for neutrons of different wavelength: (a) $\lambda_{\mathrm{n}}=0.410 \mathrm{~nm} ;(b) \lambda_{\mathrm{n}}=0.243 \mathrm{~nm}$; $(c) \lambda_{\mathrm{n}}=0.471 \mathrm{~nm}$.

the FWHM decreases by a factor of three to four times as the $H w$ increased by a factor of two. This effect is described well by the expression

$$
\mathrm{FWHM}=\chi(H w)^{-1},
$$

where $\chi$ is a parameter depending on the neutron wavelength. To study this effect in more detail, additional investigations are needed, for example, measuring the FWHM of doublecrystal rocking curves as a function of $\mathrm{Hw}$ at the centre of the Borrmann fan.

\section{Conclusions}

For describing the diffraction in single perfect crystals the theory of dynamical neutron scattering has been employed. This approach considers the neutron wave pattern propagating in the periodic potential of an ideal lattice, and a variety of features are explained by this theory, including an oscillating form for the distribution of the diffracted intensities within the Borrmann fan in the case of Laue diffraction. One of the most important dynamical diffraction effects is the strongly limited intensity diffracted by an ideal crystal in the Bragg position. However, the interference effects disappear very quickly if the translation symmetry in a perfect crystal is violated due to any disturbance (static deformation strain, low-frequency sound excitations etc.) and the Bragg-reflected intensity increases. In our work it is shown that even a very sharply rising diffraction intensity at the centre of the Borr- mann fan $\left(I_{s} / I_{0} \geq 10\right)$ can be described quantitatively using the dynamical diffraction model for the neutron propagating in a thick crystal as a spherical wave.

The ultrasonic lattice vibrations create a 'sound' strain (deformation), $\delta_{s}=k_{s} w$. For $|H w| \simeq 5$ and an AW frequency $v_{s}=41.3 \mathrm{MHz}$ the relative lattice deformation $\delta_{s}$ can reach the rather high level of $\sim 2 \times 10^{-5}$. Nevertheless, no transition to kinematic scattering occurs, which could be explained by the creation of a new periodical potential (superlattice) in the ideal crystal by a standing wave, which determines not only spatial interference effects at small $H w$ values but the features of diffraction at large $H w$ too.

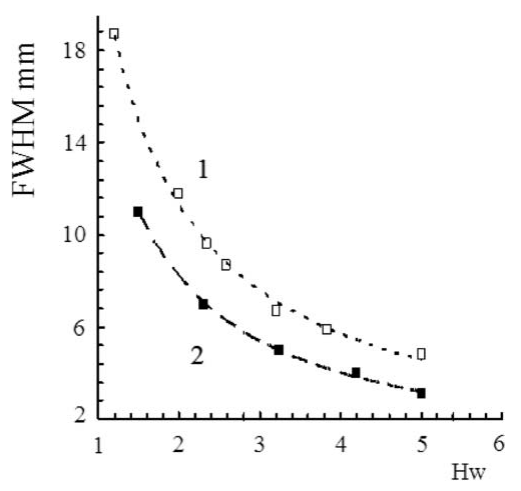

Figure 10

FWHM of the central peaks versus AW amplitude for neutrons of different wavelengths: (1) $\lambda_{\mathrm{n}}=0.410 \mathrm{~nm}$, (2) $\lambda_{\mathrm{n}}=0.243 \mathrm{~nm}$. 
The authors thank Professor V. A. Somenkov (Kurchatov Centre, Moscow, Russia) for the unique Ge sample used in this work and Dr M. Strobl (BENSC, Berlin) for help with the measurements. The research at HZB BENSC (Germany) was supported by EU programme contract No. NM13 II-1523.

\section{References}

Abov, Yu. G., Elyutin, N. O. \& Tyulyusov, A. N. (2002). Phys. At. Nucl. 65, 1933-1979.

Entin, I. (1979). Sov. Phys. JETP, 50, 110-113.

Entin, I. \& Puchkova, I. (1984). Sov. Phys. Solid State, 26, 1995-1998.

Guigay, J. P., Mikula, P., Hock, R., Baruchel, J. \& Waintal, A. (1990). Acta Cryst. A46, 821-826.

Hock, R., Liss, K. D., Magerl, A., Randl, O. G. \& Remhof, A. (1998). J. Appl. Cryst. 31, 223-234.

Hock, R., Vogt, T., Kulda, J., Mursic, Z., Fuess, H. \& Magerl, A. (1993). Z. Phys. B, 90, 143-149.

Iolin, E. \& Entin, I. (1983). Sov. Phys. JETP, 58, 985-989.

Iolin, E., Farago, B., Mezei, F., Raitman, E. \& Rusevich, L. (1998). Physica B, 241-243, 1213-1215.

Iolin, E., Raitman, E., Kuvaldin, B. \& Zolotoyabko, E. (1988). Sov. Phys. JETP, 67, 989-997.

Iolin, E. M., Rusevich, L. L. \& Vrana, M. (1996). Phys. Status Solidi $B, \mathbf{1 9 5}, 21-35$.
Iolin, E., Zolotoyabko, E., Raitman, E., Kuvaldin, B. \& Gavrilov, V. (1986). Sov. Phys. JETP, 64, 1267-1271.

Kato, N. (1969). Acta Cryst. A25, 119-128.

Kato, N. \& Ando, Y. (1966). J. Phys. Soc. Jpn, 21, 964-968.

Kato, N. \& Lang, A. R. (1959). Acta Cryst. 12, 787-794.

Kulda, J., Vrána, M. \& Mikula, P. (1988). Physica B, 151, 122-129.

Kuvaldin, B. (1991). Personal communication.

McSkimin, H. J. (1950). J. Acoust. Soc. Am. 22, 413.

Michalec, R., Mikula, P., Vrána, M., Kulda, J., Chalupa, B. \& Sedláková, L. (1988). Physica B, 151, 113-121.

Mikula, P., Lukáš, P. \& Kulda, J. (1992). Acta Cryst. A48, 72-73.

Raitman, E., Gavrilov, V., Mjasischev, D. \& Brezgunov, M. (2009). J. Surf. Inv. 3, 902-907.

Raitman, E., Gavrilov, V., Mjasischev, D., Hoser, A. \& Stusser, N. (2010). Nucl. Instrum. Methods Phys. Res. B, 268, 3411-3414.

Rauch, H. \& Petrashek, D. (1979). Topics in Current Physics, Vol. 6, edited by H. Dachs, pp. 303-351. Berlin, Heidelberg, New York: Springer-Verlag.

Shull, C. G. (1968). Phys. Rev. Lett. 21, 1585-1589.

Shull, C. G. (1973). J. Appl. Cryst. 6, 257-266.

Takagi, S. (1962). Acta Cryst. 15, 1311-1312.

Taupin, D. (1961). Bull. Soc. Fr. Miner. Cristallogr. 84, 51-53.

Truell, R., Elbaum, Ch. \& Chick, B. (1972). Ultrasound Methods in Solid-State Physics, edited by I. Michailov, p. 349. Moscow: Mir.

Zeilinger, A., Shull, C. G., Arthur, J. \& Horne, M. A. (1983). Phys. Rev. A, 28, 487-489. 